\title{
Inverse Spectropolarimetric Modelling of Hot Star Wind Structure and Variability
}

\author{
John C. Brown ${ }^{1}$, Richard Ignace ${ }^{1}$, and M. Piana ${ }^{2}$ \\ 1 Dept of Physics and Astronomy, University of Glasgow, G12 8QQ, UK \\ 2 INFN, Universita di Genova, Genova, Italy
}

\section{Introduction}

Merits and limitations are discussed of using an inferential inverse, as opposed to the usual model-fitting, approach to diagnose stellar wind structure. We aspire to encourage the stellar wind community to use inversion rather than forward modelling by making it clear what inversion means, when and why it is valuable, and by giving examples of successful applications.

A sub-discipline of astrophysics advances beyond the discovery era as the quality of data moves from single numbers (flux, colour, size) to well measured functions or data strings $g(y)$ (e.g., light curves or spectra where $g$ is flux and $y$ is time or wavelength). As the precision $\delta g / g$ and the resolution $\delta y / y$ improve, we progress from data gathering and qualitative description to quantitative modelling in terms of some relevant source model function $f(x)$, describing source "structure" in some sense. In general $g(y)$ and $f(x)$ do not correspond one-to-one but rather $f$ maps to $g$ in a "convolved" (sometimes complex and non-linear) way through the radiation processes, while $f$ itself may be a combination of important source properties. Here we consider only the simplest situation (though our arguments can be generalised) where the relationship is of the linear integral form

$$
g(y)=\mathcal{K}[f(x) ; y]=\int_{D(y)} f(x) K(x, y) d x,
$$

where the operator function $\mathcal{K}$ represents the emission physics which we assume known. The diagnostic problem is to determine as much as possible about $f(x)$ within the noise and resolution limits of the data $g(y)$ and the smearing effect of $K(x, y)$.

\section{Forward and Inverse Approaches}

The forward approach to (1) is to formulate some parametric empirical or (deductive) physical "model" for $f(x)$ and adjust parameters to yield the best fit to $g(y)$. This is the approach mostly, and often solely, used in astrophysics. Its principal merits are simplicity and the ability to deal readily with problems where $\mathcal{K}$ is complex or even where (1) is nonlinear, such as in full radiative transfer. When data are of moderate accuracy and resolution, it is 
the sensible approach. It suffers, however, from insufficiently recognised dangers of non-uniqueness of the $f(x)$ it yields. Though a "best" fit parametric $f$ can always be found, a wide range of $f$ 's may yield a statistically acceptable fit, a possibility which should always be assessed by finding the confidence band of $f$ within the errors. Moreover, the parametric form adopted for $f$ may be restrictive, thereby giving a narrow parameter confidence interval on $f(x)$ that seems very good, whereas a wholly different parametric form of $f$ might fit the data equally well. In Popperian terms, the data is not providing a critical test of the model hypothesis, goodness of fit being highly misleading (Craig and Brown 1986).

In the inverse approach, no parametric restriction is placed on $f(x)$ but rather we invert (1) to infer a model $f$ inductively from $g$ or, formally

$$
f(x)=\mathcal{K}^{-1}[g(y) ; x],
$$

though an explicit form for $\mathcal{K}^{-1}$ may not exist. In practice $g$ is measured as a discrete data vector $\mathbf{g}$ and $f$ is similarly represented by $\mathbf{f}$ while $\mathcal{K}$ is replaced by a matrix $K$ so that (1) and (2) become

$$
\mathrm{g}=\mathrm{Kf} ; \mathbf{f}=\mathbf{K}^{-1} \mathbf{g} .
$$

This inverse formulation is valuable first because (2) yields an unrestricted solution for $f(x)$ from $g(y)$ alone, based solely on data rather than assumptions (other than in the adopted form for the radiation process $K$ ). Second, the non-uniqueness in $f$ becomes fully transparent since, if $g$ is not model sensitive, $\mathcal{K}$ is correspondingly near singular and $\mathcal{K}^{-1}$ produces large magnification of errors in $g$ when data are inverted to yield $f$, whose spread is then explicit.

These advantages are only achievable when data are of sufficient resolution and accuracy for (2) and (3) to be really meaningful. This situation is reached only at quite advanced stages of astrophysical studies. For example, the inversion of the bremsstrahlung spectral problem by Brown (1971) (when data had typically $10 \%$ accuracy and $30 \%$ resolution) only recently became tenable via large, high resolution, Ge detectors with accuracy and resolution of order 1\% (cf., Thompson et al 1991). The magnified errors in $f$ caused by $\mathcal{K}^{-1}$ must be suppressed by incorporating some a priori smoothing condition on the inversion (cf., Craig and Brown 1986), but this is under our explicit control and not hidden in parametric assumptions. The main limitation is that simplifying assumptions may have to be made to formulate the inverse problem explicitly.

\section{Examples}

Here we simply list a few examples of stellar wind structure problems in which the inverse approach has been shown to be valuable. 
a) Polarisation and Stellar Occultation of Non-axisymmetric Disks: Fox and Brown (1991) showed how the 2-D density structure of rotating disk features such as CIRs could be inferred from time variability of the Stokes Parameters of scattered stellar continuum emission.

b) Spectropolarimetric Line Profiles of Disk Scattered Stellar Lines: Brown and Wood (1994) inverted the equations for the profiles of scattered stellar lines to yield the velocity structure of rotating or expanding disks. The complicating effect of thermal smearing of these profiles (Wood and Bjorkman 1995) can be dealt with as an extra convolution.

c) Polarimetry and Spectrometry of Episodic Mass Loss: Brown and Wood (1992), Calvini et al (1995), and Piana et al (1995) derived variations in rate and oblateness of stellar mass loss transients by deconvolving these from light curves of continuum scattering polarisation and absorption line strength.

d) Mean Wind Velocity Structure $v(r)$ from Line Profiles: Shapes and intensities of wind emission profiles depend on $v(r)$. Brown et al (1997) and Ignace et al $(1998 \mathrm{a}, \mathrm{b})$ obtained line profile inversions in several cases, including stellar occultation which improves the inversion.

With ever better stellar wind data in the temporal and spectral domains, the value of inverse diagnostic techniques over model fitting has reached the stage where they should be more fully exploited.

Acknowledgements-This work is supported by PPARC Standard and Visitor Grants and by a NATO Collaborative Research Grant.

\section{References}

Brown, J.C. (1971): Solar Phys. 18, 489-502

Brown, J.C., Wood, K. (1992): Astron. Astrophys. 265, 663-668

Brown, J.C., Wood, K. (1994): Astron. Astrophys. 290, 634-638

Brown, J.C., Richardson, L.L., Ignace, R., Cassinelli, J.P. (1997): Astron. Astrophys. 325, 677-684

Calvini, P., Bertero, M., Brown, J.C (1995): Astron. Astrophys. 309, 235-244

Craig, I.J.D., Brown, J.C. (1986): Inverse Problems in Astronomy (Hilger, Bristol) Fox, G.K., Brown, J.C. (1991): Astrophys.J. 375, 300-313

Ignace, R., Brown, J.C., Richardson, L.L., Cassinelli, J.P. (1998a): Astron. Astrophys. 330, 253-264

Ignace, R., Brown, J.C., Milne, J.E., Cassinelli, J.P. (1998b): Astron. Astrophys. 337, 223-232

Piana, M., Brown, J.C., Calvini, P. (1995): Inverse Problems 11, 961-973

Thompson, A.M., Brown, J.C., Craig, I.J.D., Fulber, C. (1992): Astron. Astrophys. 265, 278-288

Wood, K., Bjorkman, J.E. (1995): Astrophys. J., 443, 348-362 


\section{Discussion}

A. Sapar: The inverse solution of the radiative transfer equation in its integral form belongs to the class of ill-posed problems in mathematics and its application is in the present case limited to optically thin media. What are the maximum values of optical depth for which the method can be applied successfully?

J. Brown: Most inverse problems in radiative transfer are (because of the $e^{-\tau}$ factor) like the Laplace transform, whose inversion is indeed very illposed. Even severely ill-posed problems can be solved by regularisation methods, so the issue is not really that of a maximum optical depth but rather what is the best resolution achievable for the source function. For the Laplace problem this is only 2 or 3 points per decade for typical data accuracies.

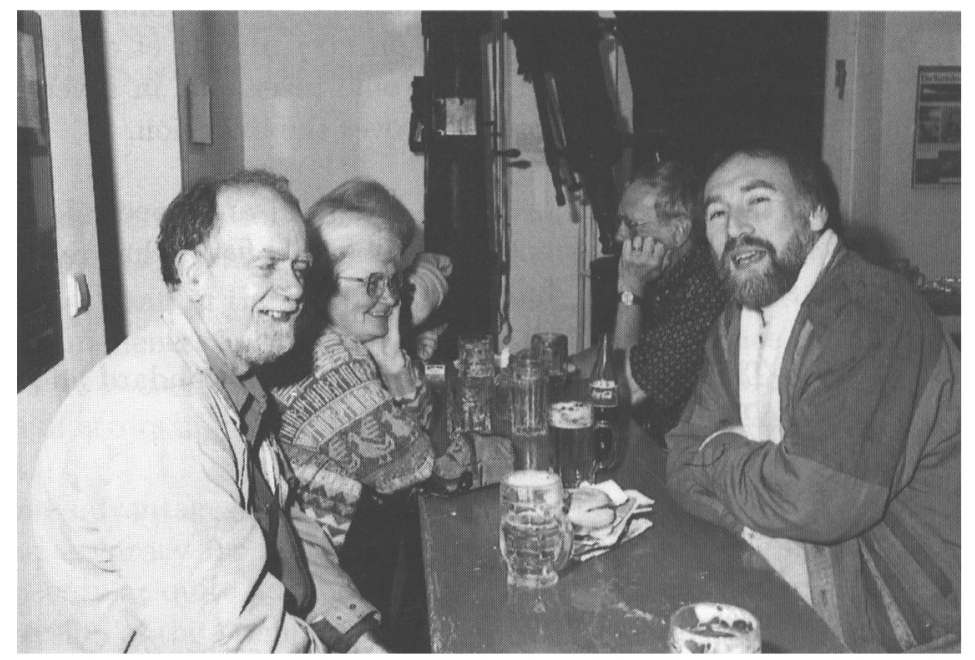

John Brown and Huib Henrichs (Joseph Cassinelli in the background) 